\title{
Prevalence of Vaginal HPV Infection Among Adolescent Girls in Jos, North-Central Nigeria
}

Nanma T. Cosmas

University of Jos

Lohya Nimzing

University of Jos

Daniel Egah

University of Jos

Ayo Famooto

Institute of Human Virology

Sally N. Adebamowo

University of Maryland, School of Medicine

Clement A. Adebamowo ( $\nabla$ cadebamowo@som.umaryland.edu )

University of Maryland, School of Medicine

\section{Research Article}

Keywords: Age-specific, Prevalence, HPV, Adolescent girls, HPV vaccine introduction, Jos Nigeria

Posted Date: August 5th, 2021

DOI: https://doi.org/10.21203/rs.3.rs-749817/v1

License: (c) (i) This work is licensed under a Creative Commons Attribution 4.0 International License.

Read Full License 


\section{Abstract}

Purpose: Knowledge of the prevalence of HPV infection among adolescents is essential to determining the best age for introduction of HPV vaccine, monitoring vaccine efficacy and giving insight into determinants of persistent high-risk HPV infection, a necessary cause of cervical cancer. Yet, there's been limited studies in low-and-middle-income countries.

Methods: In this cross-sectional study, we randomly selected 205 girls, aged 9-20 years, from 10 schools. We obtained informed consent and assent, collected data and trained participants who self-collected vaginal samples using swab stick. We genotyped HPV using $\mathrm{SPF}_{10}-\mathrm{DEIA} / \mathrm{LiPA}_{25}$ and analyzed data using Stata $14 \circledast$.

Results: The mean (SD) age of the girls was 14.9 (2.3) years. We found HPV in $13.2 \%$ of vaginal swabs. The earliest age at which anyHPV and hrHPV infections were detected was 10 and 12 years respectively. The prevalence of any HPV peaked at 16 and 17 years, hrHPV at 16 years, IrHPV at 17 and 18 years and multiple hrHPV 18 years of age. The prevalence of hrHPV infection was $1.5 \%$ among the $9-12$ years age group, $2.9 \%$ among $13-16$ years and $3.4 \%$ among $17-20$ years old. The commonest hrHPV types detected were $52(3.9 \%), 18(1.5 \%)$ and 51 (2.4\%). The most common IrHPV types was $6(2.9 \%)$.

Conclusion: The prevalence of HPV infection in these urbanized young girls in Nigeria is high and commences after 9 years of age. HPV vaccination in this population should start at 9 years of age or younger to prevent establishment of persistent HPV infection.

\section{Introduction}

Human papillomavirus (HPV) infection is the commonest oncogenic virus infection in the world. It is associated with cancers of the cervix, anus, vagina, oropharynx, vulva, oral cavity, penis and larynx[1,2]. In 2018, there were estimated 569,000 new cases of cervical cancer and 311,000 deaths worldwide[3]. Individuals are exposed to HPV infection at onset of sexual maturation and acquisition of new sexual partners $[4,5]$. Globally, data have shown that HPV prevalence is highest among young women of less than 25 years[6]. Although the prevalence and type distribution of HPV vary across geographical regions, the infection among adolescent girls usually peaks at around 15 and 16 years of age and coincides with the onset of sexual activities[5, 7]. Among older women, several studies have shown that HPV has a bimodal peak with the first peak seen among women younger than 30 years of age and the second one among older women greater than 45 years of age[8, 9]. Data are however scarce on the earliest age at which HPV is detected among adolescent girls and the exact age at which the first peak of HPV infection is observed.

HPV vaccination has been shown to be efficacious for prevention of infection and associated diseases[10] The vaccine induces stronger immunologic response when administered at a young age. Therefore, to prevent establishment of HPV infections and reduce the risk of associated diseases, the WHO recommends vaccination of girls, age 9-13 years, before they become sexually active[11]. The U.S. 
Food and Drug Administration (FDA) has licensed several prophylactic HPV vaccines. Of these, Gardasil (B) 9, a nanovalent vaccine that covers HPV types $6,11,16,18,31,33,45,52$, and 58 , licensed for use in females aged 9 to 45 years is the most widely used. There are few studies of the epidemiology of HPV infection in young girls in low- and middle-income countries to ascertain the most appropriate age range for HPV vaccination.

Substantial progress has been made in reducing HPV prevalence in developed countries like the USA[12] and Australia[13]. By 2018, 80 countries have included HPV vaccine in their national immunization programme for girls[14]. HPV infection remains of considerable public health significance, particularly in sub-Saharan Africa (SSA) where most countries are yet to implement nationwide HPV vaccination strategies[15].

While there has been several studies of the prevalence of HPV infection among HIV positive and negative women in Nigeria[16, 17], data are scarce regarding infection among adolescent girls. In the study we report the first study of the epidemiology of HPV infection in young girls in Nigeria.

\section{Methodology}

\section{Participant enrolment and sample collection}

We randomly selected 15 out of 300 registered high schools in Jos, North Central Nigeria for this study and received permission to conduct the study in 10 (66.7\%) of the schools. Between May and November 2016, we contacted 700 female students and their parents, of whom 232 girls and their parents agreed to participate in the study giving a $33.1 \%$ response rate. We obtained consent from the parents of girls younger than 18 years of age and assent from the girls. Older girls gave consent in their self-cognizance.

We conducted face-to-face interview with the girls in company of female nurses or researchers using structured questionnaires that covered socioeconomic factors, family characteristics, gynecologic history, sexual hygiene and practices. Questionnaire designs were guided by the research questions and literature review. The questionnaires were pre-tested on 10 individuals to evaluate their performance and corrected as required.

Prior to sample collection, we trained study participants on how to self-obtain vaginal samples using sterile swab sticks - a small cotton swab on a wooden handle packaged in an individual reclosable plastic pack. After the training, each girl was given 2 swab sticks and instructed to:

- Remove the swab from the reclosable pack.

- Gently insert the cotton swab into the vagina while squatting or standing with legs apart.

- Gently turn the swab three times and remove.

- Replace the swab in the plastic pack.

- Repeat this procedure with the second swab stick. 
- Swabs were inserted for about $3 \mathrm{~cm}$ and NC was always present to guide the girls

The first collected vulvo-vaginal swab was immediately stored on ice pack in a cooler until transported to the Plateau State Human Virology Research Centre (PLASVIREC) sample repository for storage at $-80^{\circ} \mathrm{C}$, usually within three hours of collection. The second swab was immediately applied on Hydrion $\mathrm{pH}$ test paper (pH 2.8-4.6, 4.5-6.1 \& 5.5-8.0) (Micro Essential Lab. Inc. New York) the colour change was compared with the provided standards.

\section{HPV detection and genotyping}

Collected samples were transported to the African Collaborative Centre for Microbiome and Genomics Research (ACCME), Institute of Human Virology, Abuja, Nigeria for HPV detection and genotyping. To prepare the vulvo-vaginal swab for DNA extraction, 1 millilitre of PBS was added to the dry swabs inside the cryovials and vortexed to dislodge the cells from the cotton wool.

DNA extraction was carried out using Cador ${ }^{\circledR}$ Pathogen 96 QIAcube ${ }^{\circledR}$ HT Kit (Qiagen, Germany) on QIAcube HT (QIAGEN, Germany). The DNA was amplified and HPV detected using SPF 10 (DDL Diagnostic Laboratories, The Netherlands) which simultaneously detects HPV types $2,3,4,5,6,7,8,11,13,14,16$, $18,20,26,27,28,30,31,32,33,34,35,37,39,40,42,43,44,45,51,52,53,54,55$ (re-classified as a subtype of HPV44), 56, 57, 58, 59, 61, 62, 64 (re-classified as a subtype of HPV34), 65, 66, 67, 68, 69, 70, $71,72,73,74,75,76,81,82,83,84,85,86,87,89,90,91,95,97,102,106,114$ and 115. Positive samples were tested with reverse hybridization line probe assay $\left(\mathrm{LiPA}_{25}\right)$ which can simultaneously identify 25 HPV types $(6,11,16,18,31,33,34,35,39,40,42,43,44,45,51,52,53,54,56,58,59,66,68-73$, 70, and 74) according to the manufacturer's (DDL Laboratories, Netherlands) instructions. This is a highly sensitive broad-spectrum PCR-based assay for qualitative detection of HPV and suitable for epidemiological studies.

\section{Ethical approval and informed consent}

The study protocol was approved by the institutional review board of the Jos University Teaching Hospital (JUTH).

\section{Data management and Statistical analysis}

We entered data into REDCap electronic database and analyzed using Stata version 14 (StataCorp, College Station, Texas USA). High-risk HPV types were defined as types 16, 18, 26, 31, 33, 35, 39, 45, 51, $52,53,56,58,59,66,88,73$ and 82 , including the probable hrHPV types and low-risk types were defined as types $6,11,40,42,43,44,54,61,68 / 73,70,72,74,81$ and CP6108[18]. Univariate analysis was done using frequency distribution and proportion for categorical variables and descriptive statistics for 
continuous variables. Bivariate analysis to test for association was done using chi square or fisher's exact test for categorical variables and t-test for continuous variables. A p-value $<0.20$ was used as a criterion for the inclusion of variables in the multivariable analysis. We set a $p$-value $<0.05$ as statistically significant

\section{Results}

\section{Participants' Characteristics}

We excluded 27 participants (11.6\%) because of inability to collect vulvo-vaginal swab leaving 205 participants. The range and mean age (SD) of the excluded girls were 11-20 and 14.7 (1.97) years while that of the remaining study participants were 9-20 and 14.9 (2.34) years ( $p$-value 0.67). Most of the excluded participants $(17,63.0 \%)$ were from public schools while the remaining $37.0 \%$ were from private schools. About half of the remaining participants, $(104 / 205,50.7 \%)$, were recruited from public schools while $49.3 \%(101 / 205)$ were from private schools ( $p$-value 0.24$)$. There was no significant difference in the characteristics of the girls who were unable to collect their samples and were therefore excluded from the study and those who remained in the study.

Most, $70.7 \%$, of the girls in this study had attained menarche and $9.27 \%$ reported positive history of sexual intercourse. The range and mean (SD) age at first sexual intercourse were 6-18 and 13.3 (3.58) years. Most of the girls (57.9\%) experienced their first sexual intercourse before 15 years of age and $15.8 \%$ before the age of 10 years. Fourteen girls $(6.8 \%)$ reported having one lifetime number of sexual partners while $3(1.5 \%)$ reported 2 sexual partners. One girl each reported 3 and 4 sexual partners. Of the 19 girls who were sexually active, $11(57.9 \%)$ reported history of forced sex without consent. Some $16.6 \%$ of the girls douche, and of these $14.7 \%$ reported ever having sex while $85.3 \%$ did not report history of sexual contact. There was no statistically significant association between vaginal douching and sexual contact $(p=0.23)$.

The mean (SD) vaginal $\mathrm{pH}$ of the girls in this study was $4.8(0.47)$ and the median was 4.5. The vaginal $\mathrm{pH}$ was greater than 4.5 in $45.8 \%$ of the girls while $3.48 \%$ had vaginal $\mathrm{pH}$ less than 4.5 . Approximately $60.0 \%$ of the participants with vaginal $\mathrm{pH}$ of 4.5 reported ever haing sex and $35.3 \%$ reported vaginal douching practice, while $36.8 \%$ and $61.8 \%$ of those with vaginal $\mathrm{pH}>4.5$ reported ever having sex and vaginal douching practice respectively. Of those with vaginal $\mathrm{pH}<4.5,5.3 \%$ had ever had sex and $2.9 \%$ reported vaginal douching practice. The associations between vaginal $\mathrm{pH}$ and history of sexual contact and vaginal douching practice were not statistically significant. Seven participants (3.4\%) reported history of masturbation and the earliest year for initiation of the act was 9 years of age. (Table 1 ).

\section{Group Specific Prevalence of HPV}

The prevalence of any HPV infection in this study was $13.2 \%$, while that of hrHPV was $7.8 \%$, IrHPV was $7.3 \%$, mixed high and low risk HPV was $2.0 \%$ and multiple hrHPV was $2.9 \%$. The age-specific and age 
group prevalence of HPV among study participants are shown in Figure 1. The first instance of any type of HPV infection among the girls in this study was at 10 years of age and it was a low-risk HPV infection. The first instance of high-risk HPV infection was observed at 12 years of age. The peak point age prevalence of anyHPV, hrHPV, IrHPV and multiple hrHPV was 16 years of age. At this age, the prevalence of anyHPV was $6.8 \%$, while that of hrHPV was $4.9 \%$, IrHPV was $2.6 \%$ and multiple hrHPV was $2.4 \%$. The prevalence of anyHPV, hrHPV, IrHPV and multiple hrHPV was highest in the 13-16 age group. The prevalence of anyHPV infection was $4.9 \%$ among 9-12 years age group, 15.1\% among $13-16$ years and 9.3\% among 17-20 years old while that of hrHPV infection was $3.4 \%$ among $9-12$ years age group, $7.8 \%$ among $13-16$ years and $5.4 \%$ among $17-20$ years old. The prevalence of IrHPV was $2.0 \%$ in the $9-12$ years age group, $8.8 \%$ in $13-16$ years age group and $5.4 \%$ in the $17-20$ years age group while the prevalence of multiple HPV infections is $1.5 \%$ in the $9-12$ years age group, $3.4 \%$ in $13-16$ years age group and $2.4 \%$ in the $17-20$ years age group.

\section{Type Specific Prevalence of HPV}

The commonest HPV type found in this study population was HPV unknown type $11.2 \%$, suggesting substantial prevalence of HPV types beyond those detected using $\mathrm{SPF}_{10} \mathrm{LiPA}_{25}$ in this study population. We detected nine hrHPV types and the commonest hrHPV types were 52 (10.2\%), 18 (6.4\%) and 51 (4.5\%) (Figure 2). The other hrHPV types detected in this study were 58 (2.0\%), 66 (1.5\%), 56 (1.5\%), 45 (1.0\%), $31(0.5 \%)$ and $35(0.5 \%)$. We did not detect any HPV type 16 in this study sample. The most common IrHPV type we identified was type $6(3.4 \%)$ followed by type $44(1.0 \%)$. Other IrHPV types detected were types $68 / 73(0.5 \%), 70(0.5 \%)$ and $74(0.5)$. We did not detect IrHPV type 11 among this cohort of adolescent girls.

The age at first detection of HPV types $18,45,66,6$, and 74 was 16 years, while HPV types 51,52 , and 58 was 12 years. HPV types 31,44 and $68 / 74$ were first detected at 19,15 and 17 years respectively.

\section{Risk factors for HPV infection}

Other risk factors for HPV infection were examined and presented on table 2. Only number of sexual partners was independently associated with HPV detection. In multivariable analysis for risk of anyHPV that included age and categories of wealth index, only history of ever having sex was statistically significant (OR $(95 \% \mathrm{Cl}): 3.10(1.03-9.30)$, $p$-value $=0.04)$ There was no statistically significant associations between low or high risk HPV and any other variable in multivariable analyses.

\section{Discussion}

Previous studies in Nigeria have reported the prevalence of HPV infection in the general population of women and also among HIV positive women[16, 17]. Up to now, far too little attention has been paid to the epidemiology of HPV infection among adolescent girls, who are the target group for HPV vaccination. 
We presented here one of the first studies to focus specifically on the prevalence and type distribution of HPV infection among adolescent girls in Nigeria.

The overall prevalence of HPV found in this population of adolescent females irrespective of the sample type was $29.3 \%$. Globally, the overall prevalence of HPV infection varies according to geographical regions. This is slightly higher than the $24.5 \%$ finding among adolescent girls age 14-19 years in the USA before the introduction of HPV vaccination[19]. A studies in India[20] has also reported lower HPV prevalence than the finding of this study among adolescent girls. However, the prevalence of HPV in this study is lower than those reported from South Africa and Burkina Faso, which ranged between $38 \%-67 \%$ $[21,22]$.

Age is an important determinant of HPV vaccination. The WHO recommends vaccine administration as early as age 9 years targeting girls before the onset of sexual activity. In this study, the earliest age at which anyHPV and hrHPV infections were detected in either vulvo-vaginal or urine samples were 10 and 11 years respectively. In this study HPV Infection peaked at age 16 years and declined with increasing age. This corroborates with previous findings that adolescent girls have higher exposure to HPV infection[23] and could indicate exposures to sexual activities, which we found to be high around this age group. This also confirmed the finding that the peak age of HPV infection occurs among younger people less than 25 years of age[6]. The WHO target group for the primary prevention of HPV infection is age 914 years, prior to sexual debut. Introduction of HPV vaccine in the population of adolescent girls in SSA will be of great benefit among 9-14 years old before infection peak, which was 16 years in this study. Early intervention through vaccine introduction and achieving of high vaccine coverage would reduce the risk of HPV infection among girls, and evidence from Australian data have shown a reduction of genital warts in both vaccinated women and heterosexual men[24]. Although HPV infection prevalence peaked at age 16 years, catch-up vaccination of girls greater or equal to 15 years would also be beneficial in the prevention of other HPV types in the vaccine for which these girls are still naive to.

The high prevalence of HPV recorded in this study is of public health concern and underscores the importance of introduction of HPV in Nigeria for the prevention of HPV infection and associated malignancies. Although more than $90 \%$ of all infection with any HPV resolve without treatment within 2 years approximately $10 \%$ of infection fail to resolve, resulting in persistent infection with the virus[25]. Persistent infection with hrHPV type is the primary risk factor for the development of HPV related cancer[26].

The finding of considerably high prevalence of HPV infection in this study demonstrates the need for HPV persistence studies among adolescent girls to identify those that require monitoring. This study has given a peep hole into the requirement for further studies on HPV and other STIs, which are likely to remain undiagnosed and may impact negatively on sexual reproductive health.

HPV vaccination is best administered to HPV naïve individuals and before the onset of sexual activities[11]. The earliest age for ever had sexual contact in this study was 11 years of age. Other studies have also shown that sexual debut before age 15 years was common among young people[27, 28]. Early 
age at sexual debut has implications for hrHPV infection[29] and has also been associated with other risky sexual behaviour[30], which contribute to increase the likelihood of HPV infection persistence and progression to cancer. Based on the findings of this study, we recommend that more effort should be put in by the Nigerian Government and other stake holders to facilitate the introduction of HPV vaccine among the at risk group, especially that some of these young girls are already sexually active.

The major limitation of the study is the small sample size but given our methods, the results are likely representative of the larger population of adolescent girls in Jos.

\section{Conclusion}

To the best of our knowledge, this is the first HPV study to be conducted in Nigeria that focused mainly on primary target group for HPV vaccine. Knowledge about HPV infection prevalence among adolescent girls had provided an important gateway into HPV vaccine introduction in and vaccine success evaluation. Our findings indicate that HPV vaccination targeting 9 year olds would adequately cover the at-risk population in Nigeria. The study lays the foundation for future monitoring of vaccine efficacy and give insight into the determinants of persistent high-risk HPV infection (hrHPV) among the study population.

\section{Key Messages}

- The prevalence of HPV was found to be $13.2 \%$ among adolescent girls in Jos, North-Central Nigeris.

- The earliest age at which HPV infection was detected in vaginal samples was 11 years of age.

- The most predominant hrHPV types in vaginal swab was HPV 52.

- These findings point to the need for introduction of HPV vaccine among girls in Nigeria and should target girls at 9 years or younger.

\section{Declarations}

\section{Funding Statement:}

This was supported by Office Of The Director, National Institutes Of Health and the National Human Genome Research Institute (U54HG006947); funds through the Maryland Department of Health's Cigarette Restitution Fund Program; and the University of Maryland Greenebaum Cancer Center Support Grant (P30CA134274).

\section{Competing Interests Statement:}

We have no conflicts of interest to disclose. 


\section{Contributorship Statement:}

N.T.C., C.A.A. contributed to study design, implementation and sample/data collection; N.T.C, F.A. analyzed the biological samples; N.T.C., S.N.A., C.A.A analyzed data, generated figures and tables and contributed to data interpretation; L.N., D.E. supervised the project; N.T.C. drafted the manuscript; C.A.A. conceived the study and obtained funding for the project. All authors critically reviewed the manuscript and approved the final version for submission.

\section{Consent for publication:}

Not applicable

\section{Availability of data and materials:}

The datasets used and/or analysed during the current study available from the corresponding author on reasonable request.

\section{Abbreviations:}

Not applicable

\section{Acknowledgements:}

The authors would like to thank all the girls who participated in the study and research assistants that helped with sample and data collection. We are also grateful to the staff of African Collaborative Centre for Microbiome and Genomics Research, Institute of Human Virology, Nigeria.

\section{References}

1. Saraiya MS, Unger ER, Thompson TD, Lynch CF, Hernandez BY, Lyu CW, et al. US assessment of HPV Types in cancers: Implications for current and 9-valent HPV vaccines. Journal of the National Cancer Institute. 2015;107(6):djv086.

2. Viens LJ, Jane Henley S, Watson M, Markowitz LE, Thomas CC, Thompson TD, et al. Human papillomavirus-associated cancers - United States, 2008-2012. Morbidity and Mortality Weekly Report. 2016;65(26):661-6.

3. Ferlay JF, Ervik M, Lam F, Colombet M, Mery L, Piñeros M, et al. Global Cancer Observatory: Cancer Today [Internet]. Cancer Today. 2018. Available from: https://gco.iarc.fr/today 
4. Winer RL, Lee SK, Hughes JP, Adam DE, Kiviat NB, Koutsky LA. Genital human papillomavirus infection: Incidence and risk factors in a cohort of female university students. American Journal of Epidemiology. 2003;157(3):218-26.

5. Weaver B, Tu W, Shew M, Qadadri B, Tong Y, Denski C, et al. 2. Acquisition of First Human Papillomavirus Infection Related to First Vaginal Intercourse and Other Sexually Transmitted Infections in Adolescent Women. Journal of Adolescent Health. 2011;48(2):S11-2.

6. Smith JS, Melendy A, Rana RK, Pimenta JM. Age-Specific Prevalence of Infection with Human Papillomavirus in Females: A Global Review. Vol. 43, Journal of Adolescent Health. 2008. p. S5.e1S5.e62.

7. Wellings K, Collumbien M, Slaymaker E, Singh S, Hodges Z, Patel D, et al. Sexual behaviour in context: a global perspective. Vol. 368, Lancet. 2006. p. 1706-28.

8. Dareng EO, Adebamowo SN, Famooto A, Olawande O, Odutola MK, Olaniyan Y, et al. Prevalence and incidence of genital warts and cervical Human Papillomavirus infections in Nigerian women 11 Medical and Health Sciences 1117 Public Health and Health Services. BMC Infectious Diseases. 2019;19(1).

9. Torres-Poveda K, Ruiz-Fraga I, Madrid-Marina V, Chavez M, Richardson V. High risk HPV infection prevalence and associated cofactors: A population-based study in female ISSSTE beneficiaries attending the HPV screening and early detection of cervical cancer program. BMC Cancer. 2019;19(1).

10. Joura EA, Giuliano AR, Iversen OE, Bouchard C, Mao C, Mehlsen J, et al. A 9-valent HPV vaccine against infection and intraepithelial Neoplasia in women. Vol. 70, Obstetrical and Gynecological Survey. 2015. p. 446-8.

11. World Health Organization. WHO guidance note: comprehensive cervical cancer prevention and control: a healthier future for girls and women. WHO Guidelines Note. 2013;1-12.

12. Markowitz LE, Gee J, Chesson H, Stokley S. Ten Years of Human Papillomavirus Vaccination in the United States. Vol. 18, Academic Pediatrics. 2018. p. S3-10.

13. McGregor S, Saulo D, Brotherton J, Liu B, Phillips S, Skinner SR, et al. Decline in prevalence of human papillomavirus infection following vaccination among Australian Indigenous women, a population at higher risk of cervical cancer: The VIP-I study. Vaccine. 2018;36(29):4311-6.

14. Gallagher KE, LaMontagne DS, Watson-Jones D. Status of HPV vaccine introduction and barriers to country uptake. Vaccine. 2018;36(32):4761-7.

15. Black E, Richmond R. Prevention of Cervical Cancer in Sub-Saharan Africa: The Advantages and Challenges of HPV Vaccination. Vaccines. 2018;6(3):61.

16. Adebamowo SN, Olawande O, Famooto A, Dareng EO, Offiong R, Adebamowo CA. Persistent LowRisk and High-Risk Human Papillomavirus Infections of the Uterine Cervix in HIV-Negative and HIVPositive Women. Frontiers in Public Health. 2017;5(178).

17. Akarolo-Anthony SN, Al-Mujtaba M, Famooto AO, Dareng EO, Olaniyan OB, Offiong R, et al. HIV associated high-risk HPV infection among Nigerian women. BMC Infectious Diseases. 
2013;13(1):521.

18. Muñoz N, Bosch FX, de Sanjosé S, Herrero R, Castellsagué X, Shah K v., et al. Epidemiologic Classification of Human Papillomavirus Types Associated with Cervical Cancer. New England Journal of Medicine [Internet]. 2003;348(6):518-27. Available from:

http://www.nejm.org/doi/abs/10.1056/NEJMoa021641

19. Dunne EF, Unger ER, Sternberg M, McQuillan G, Swan DC, Patel SS, et al. Prevalence of HPV infection among females in the United States. Journal of the American Medical Association. 2007;297(8):813-9.

20. Sharma K, Kathait A, Jain A, Kujur K, Raghuwanshi S, Bharti AC, et al. Higher prevalence of human papillomavirus infection in adolescent and young adult girls belonging to different indian tribes with varied socio-Sexual lifestyle. PLoS ONE. 2015;10(5).

21. Mbulawa ZZA, Van Schalkwyk C, Hu NC, Meiring TL, Barnabas S, Dabee S, et al. High human papillomavirus (HPV) prevalence in South African adolescents and young women encourages expanded HPV vaccination campaigns. PLoS ONE. 2018;13(1):e0190166.

22. Traore IMA, Zohoncon TM, Ndo O, Djigma FW, Obiri-Yeboah D, Compaore TR, et al. Research article oncogenic human papillomavirus infection and genotype characterization among women in Orodara, western Burkina Faso. Pakistan Journal of Biological Sciences. 2016;19(7):306-11.

23. Tarkowski TAA, Koumans EHH, Sawyer M, Pierce A, Black CMM, Papp JRR, et al. Epidemiology of Human Papillomavirus Infection and Abnormal Cytologic Test Results in an Urban Adolescent Population. The Journal of Infectious Diseases. 2007 Jan 10;189(1):46-50.

24. Schiffman M, Saraiya M. Control of HPV-associated cancers with HPV vaccination. Vol. 17, The Lancet Infectious Diseases. 2017. p. 6-8.

25. Stanley MA. Epithelial cell responses to infection with human papillomavirus. Vol. 25, Clinical Microbiology Reviews. 2012. p. 215-22.

26. Plummer M, Schiffman M, Castle P, Maucort-Boulch D, Wheeler C. A 2-Year Prospective Study of Human Papillomavirus Persistence Among Women With a Cytological Diagnosis of Atypical Squamous Cells of Undetermined Significance or Low-Grade Squamous Intraepithelial Lesion. Journal of Lower Genital Tract Disease. 2009;12(2):152.

27. Durowade KA, Babatunde OA, Omokanye LO, Elegbede OE, Ayodele LM, Adewoye KR, et al. Early sexual debut: Prevalence and risk factors among secondary school students in Ido-Ekiti, Ekiti state, South-West Nigeria. African Health Sciences. 2017;17(3):614-22.

28. Peltzer K, Pengpid S. Early Sexual Debut and Associated Factors among In-school Adolescents in Six Caribbean Countries. West Indian Medical Journal. 2015;64(4):351-6.

29. Bosch FX, Lorincz A, Muñoz N, Meijer CJLM, Shah K V. The causal relation between human papillomavirus and cervical cancer. Vol. 55, Journal of Clinical Pathology. 2002. p. 244-65.

30. Krüger-Kjær S, Van den Brule AJC, Svare El, Engholm G, Sherman ME, Poll PA, et al. Different risk factor patterns for high-grade and low-grade intraepithelial lesions on the cervix among HPV-positive and HPV-negative young women. International Journal of Cancer. 1998;76(5):613-9. 


\section{Tables}

Due to technical limitations, tables are only available as a download in the Supplemental Files section.

Figures

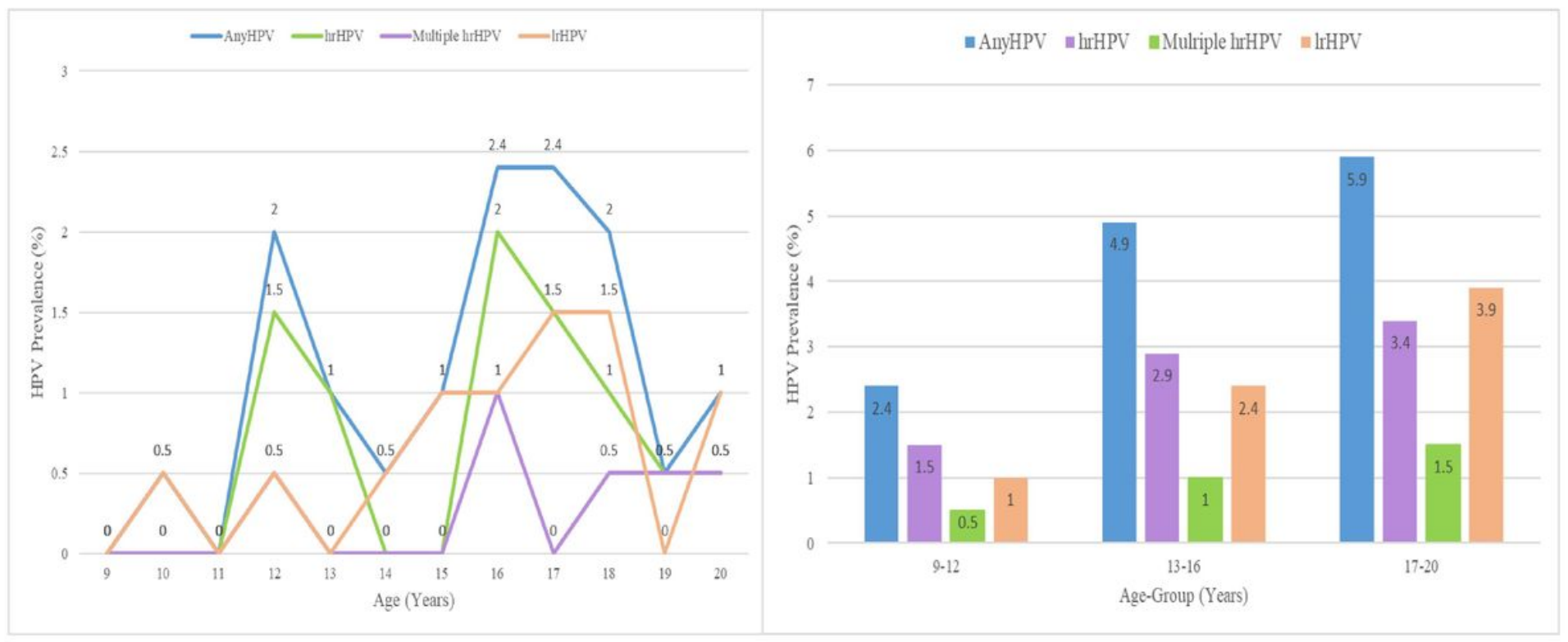

\section{Figure 1}

Age-specific and age group prevalence of HPV among girls, Jos, Nigeria. March 2017 


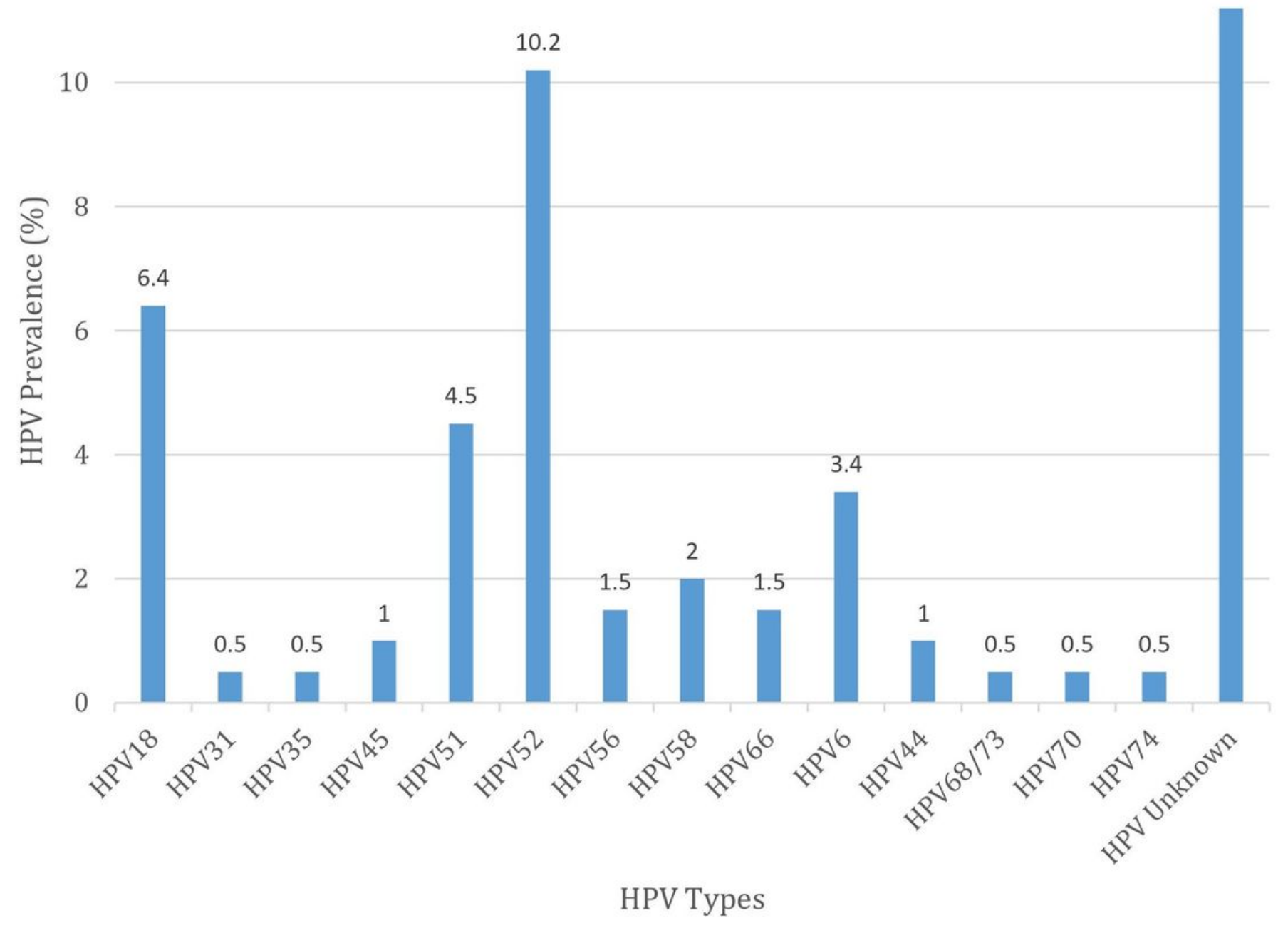

Figure 2

Prevalence of hrHPV and IrHPV types among girls in Jos, Nigeria. March 2017.

\section{Supplementary Files}

This is a list of supplementary files associated with this preprint. Click to download.

- Table1.pdf

- Table2.pdf 